\title{
Relationship between Thyroid and Kidney Function: Analysis from the Korea National Health and Nutrition Examination Survey Between 2013 and 2015
}

\author{
Sang Hyuk Kim ${ }^{a}$ Hyang Ki Min ${ }^{b}$ Sung Woo Leeb \\ a Medical Service Corps of 2nd Armored Brigade, Republic of Korea Army, Paju, South Korea; \\ ${ }^{b}$ Department of Internal Medicine, Nowon Eulji Medical Center, Eulji University, Seoul, \\ South Korea
}

\section{Keywords}

Thyroid function · Renal function · Thyroid stimulating hormone · Free thyroxine

\begin{abstract}
Introduction: Thyroid function is evaluated by thyroid stimulating hormone (TSH) and free thyroxine (fT4). Although many studies have indicated an intimate relationship between thyroid hormones and kidney functions, reports about the simultaneous evaluation of TSH and fT4 are rare. Objective: We aimed to analyze the association between TSH and kidney function, with emphasis on a potential nonlinear relationship, and identify an independent relationship between fT4 and kidney function. Methods: We reviewed the data of 7,061 subjects in the Korea National Health and Nutrition Examination Surveys who were randomly subsampled for thyroid function evaluation between 2013 and 2015. A total of 5,578 subjects were included in the final analysis, after excluding people $<18$ years old, and those with a short fasting time, abnormal fT4 levels, and thyroid disease or related medications. Creatininebased estimated glomerular filtration rate (eGFR) was used to define kidney function. Results: A $1 \mathrm{mmol} / \mathrm{L}$ increase of logarithmic TSH was associated with decreased eGFR ( $\beta$ : $-1.8 ; 95 \% \mathrm{Cl}$ -2.3 to $-1.2 ; p<0.001$ ), according to multivariate linear regression analysis. On the multivariate generalized additive model plot, TSH demonstrated an L-shaped relationship with eGFR, showing a steeper slope for $0-4 \mathrm{mIU} / \mathrm{L}$ of TSH. A $1 \mu \mathrm{g} / \mathrm{dL}$ increase of fT4 was also associated with decreased eGFR ( $\beta$ : $-7.0 ; 95 \% \mathrm{Cl}-0.94$ to $-4.7 ; p<0.001)$ on the multivariate linear regression analysis; this association was reversed after adjusting for age. On the mediation analysis, the indirect effect via age and direct effect per $1 \mu \mathrm{g} / \mathrm{dL}$ increase of fT4 on eGFR was 9.9 (8.1 to $11.7, p<0.001)$ and $-7.1(-9.3$ to $-4.8, p<0.001)$, respectively. Conclusions: Increased TSH was associated with decreased eGFR, particularly in the reference range. The direct effect of increased fT4 was decreased eGFR, which may be affected indirectly by age.
\end{abstract}




\section{Kidney \\ Blood Pressure \\ Research}

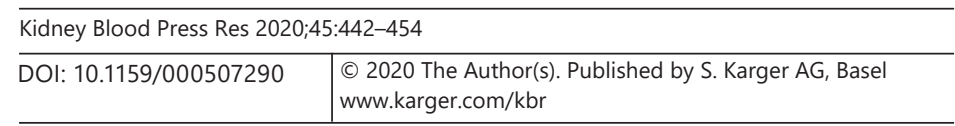

Kim et al.: Relationship Between Thyroid and Kidney Function

\section{Introduction}

Thyroid hormones affect many aspects of the kidney, including development and hemodynamics [1]. Thyroid dysfunctions should be properly and promptly treated due to the effects of thyroid hormones on the body metabolism [2,3]. Subclinical hypothyroidism (SCH) is common disease and thyroid dysfunction; there is still controversy regarding over- and undertreatment of this disease [4]. SCH is defined when the thyroid stimulating hormone (TSH) level is increased and the free thyroxine (fT4) level is normal [5]. In routine practice, doctors and clinicians do not recommend treating nonsymptomatic SCH [6]. This may be because the TSH levels in about $60 \%$ of SCH patients with a mild elevation in TSH (4-10 $\mathrm{mIU} / \mathrm{L}$ ) become normalized without intervention [7].

Nonetheless, SCH can progress to overt hypothyroidism, especially when the TSH level $\geq 10 \mathrm{mIU} / \mathrm{L}$ [8]. Furthermore, it may be associated with diastolic heart dysfunction [9], arterial stiffness [10], diastolic hypertension [11], dyslipidemia [12], and insulin resistance [13], which ultimately increase the risk of heart failure [14, 15], cardiovascular (CV) disease, and mortality $[16,17]$. Furthermore, SCH may also have a negative effect on kidney health. Experimental studies have suggested that a deficiency in thyroid hormones may reduce the glomerular filtration rate [18] by reducing cardiac output, renal plasma flow, and sodium resorption [19]. To date, many studies have suggested that SCH was associated with reduced kidney function [20-24]. Some have even reported that an elevated TSH level, but within the reference range, was associated with chronic kidney disease (CKD) [25-27].

However, there are few studies that have examined the effects of TSH and fT4 on kidney function simultaneously [25, 28-30]. In fact, the metabolism of T4 is altered in CKD patients; deiodination of $\mathrm{T} 4$ and T4-binding plasma proteins is reduced and inhibitors of T4 binding to plasma proteins and metabolic acidosis are presented [19]. Therefore, we postulated that the simultaneous evaluation of TSH and fT4 is important, particularly for evaluating kidney function. In this study, we aimed to analyze the association between TSH and kidney function, with emphasis on a potentially nonlinear relationship, and identify an independent relationship between fT4 and kidney function, using data from the Korea National Health and Nutrition Examination Survey (KNHANES), a nationwide government-administered survey. We hypothesize that high TSH and fT4 level could affect kidney function decline.

\section{Material and Methods}

\section{Participants}

The KNHANES has been conducted periodically, since 1998, to assess the health and nutritional status of the civilian, noninstitutionalized population of Korea. Participants are selected using multistage, stratified probability sampling. This study used data from the KNHANES from 2013 to 2015. Of 29,321 candidates, 22,948 people agreed to participate (participation rate: $78.3 \%$ ). For the 22,948 subjects, thyroid function tests were performed by subsampling 2,400 subjects aged $\geq 10$ years in each survey; a total of 7,061 subjects underwent thyroid function tests during the KNHANES, from 2013 to 2015 . We excluded 897 participants who were $<18$ years old. Of the 6,164 adult participants, 127 with an overnight fasting time $<8 \mathrm{~h}, 168$ with a low fT 4 level $(<0.92 \mu \mathrm{g} / \mathrm{dL}), 193$ with a high fT4 level $(\geq 1.60 \mu \mathrm{g} /$ $\mathrm{dL}$ ), 86 with a physician diagnosis of thyroid disease or cancer, and 12 who were administered thyroid medications were also excluded. Therefore, we included 5,578 subjects for the final analysis. 


\section{Kidney \\ Blood Pressure \\ Research}

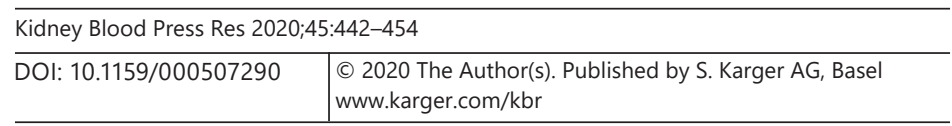

Kim et al.: Relationship Between Thyroid and Kidney Function

\section{Ethical Considerations}

The study was conducted in accordance with the Declaration of Helsinki, and full approval of the study was obtained from the Institutional Review Board (IRB) of the Korea Centers for Disease Control (IRB 2013-07CON-03-4C, 2013-12EXP-03-5C, 2015-01-02-6C). All data were fully anonymized before we accessed them. All KNHANES participants provided written informed consent to have their data used in research.

\section{Thyroid Function Test}

Thyroid function was tested using serum levels of fT4, TSH, and antithyroid peroxidase antibodies (anti-TPO Abs). Serum levels of fT4, TSH, and anti-TPO Abs were measured by an electro-chemiluminescence immunoassay (Cobas: Roche Diagnostics, Penzberg, Germany). Thyroid status was defined according to a general clinical consensus [31-34]. Subjects were diagnosed with hyperthyroidism if they had a TSH level $\leq 0.1 \mathrm{mIU} / \mathrm{L}$, euthyroidism if they had a TSH level ranging from 0.1 to $4.0 \mathrm{mIU} / \mathrm{L}$, and hypothyroidism if they had a TSH level $\geq 4.0$ $\mathrm{mIU} / \mathrm{L}$. Anti-TPO Abs levels $\geq 34 \mathrm{IU} / \mathrm{mL}$ were assumed to be positive [35].

\section{Measurements}

A standardized interview was conducted in the homes of the subjects to determine disease diagnosis. Blood pressure (BP) was measured 3 times using a standard mercury sphygmomanometer (Baumanometer Wall Unit 33 [0850]; Baum Co., Inc., Copiague, NY, USA). Participants did not smoke, sat down for at least $5 \mathrm{~min}$, and measured BP at heart level. The average of the 3 measures was used. Body mass index was calculated by dividing the weight by the square of the height $\left(\mathrm{kg} / \mathrm{m}^{2}\right)$. Waist circumference (WC) was measured the midpoint between the lower border of the rib cage and the highest point of the iliac crest, using a tape measure (Seca 200, Seca, Germany), to the nearest $0.1 \mathrm{~cm}$. Blood and urine samples were collected in the morning after $>8 \mathrm{~h}$ fasting and analyzed in a central laboratory (Neodin Medical Institute, Seoul, Korea). High-density lipoprotein cholesterol (HDL-C), triglyceride (TG), and fasting glucose (FG) levels were measured using the Hitachi 7600 Automatic Analyzer (Hitachi, Japan). Hemoglobin levels were measured using the Sodium Lauryl Sulfate hemoglobin detection method [36]. White blood cell counts were measured using flow cytometry with a semiconductor laser. The XE-2100D (2013-2014, Sysmex, Japan) and XN-9000 (2015, Sysmex, Japan) were used to measure hemoglobin and white blood cells counts. Urine iodine was measured with inductively coupled plasma-mass spectroscopy using Perkinelmer inductively coupled plasma-mass spectroscopy (Perkin Elmer, Waltham, MA, USA), while urine protein was measured with a dipstick method using the UriSys 2400 analyzer (Roche, Germany). Serum creatinine levels were measured using a Hitachi Automatic Analyzer 7600 (Hitachi, Tokyo, Japan) with the Jaffe method [37], and kidney function was calculated from the serum creatinine using the Chronic Kidney Disease Epidemiology Collaboration equation [38].

\section{Definitions}

Metabolic disorders were defined following the recommendations of the International Diabetes Federation [39]. Central obesity was defined as a WC $\geq 90 \mathrm{~cm}$ for men and WC $\geq 80$ $\mathrm{cm}$ for women. Raised BP was defined as systolic BP $\geq 130 \mathrm{~mm} \mathrm{Hg}$, diastolic BP $\geq 85 \mathrm{~mm} \mathrm{Hg}$, or treatment with antihypertensive drugs. Raised FG was defined as FG $\geq 100 \mathrm{mg} / \mathrm{dL}$, treatment with insulin or oral antidiabetic drugs, or a previous diagnosis of type 2 diabetes. Raised TG was defined as TG $\geq 150 \mathrm{mg} / \mathrm{dL}$. Reduced HDL-C level was defined as HDL-C $<40$ $\mathrm{mg} / \mathrm{dL}$ for men and HDL-C $<50 \mathrm{mg} / \mathrm{dL}$ for women or administration of specific treatments for dyslipidemia. Previous CV disease was defined by a diagnosis of stroke, myocardial infarction, or angina. Liver disease was defined by a previous diagnosis of the hepatitis B or C virus or 


\section{Kidney \\ Blood Pressure \\ Research}

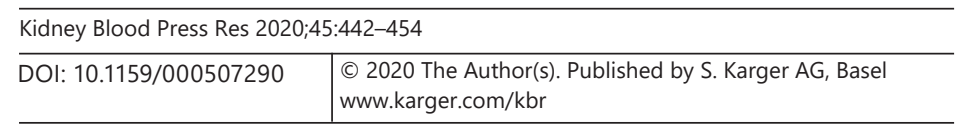

Kim et al.: Relationship Between Thyroid and Kidney Function

liver cirrhosis. Proteinuria was defined as a protein level of $1+$ or higher on the dipstick urinalysis. Regular exercise was defined as doing strenuous exercise (i.e., soccer, climbing) > 3 days a week. Regular walking was defined as walking for $>10$ min for travel, such as commuting or shopping, $>3$ days a week. High income was defined by the highest quartile of monthly personal income. Alcohol consumption was defined by consuming alcohol more than once a month within the last year.

\section{Statistical Analyses}

Histograms and Q-Q plots were constructed to evaluate the normality of continuous variables. Normally distributed continuous variables were expressed as mean (SD), while nonnormally distributed continuous variables were expressed as median (interquartile range). Categorical variables were expressed as number (percentage). The $p$ trend was analyzed for normally distributed continuous variables using a one-way analysis of variance. For nonnormally distributed continuous variables, the Jonckheere-Terpstra test was used. The CochranArmitage test was used for categorical variables. The Locally Weighted Scatter-plot Smoother (LOWESS) was used to evaluate the simple relationship between TSH, fT4, and estimated glomerular filtration rate (eGFR). Linear regression analysis was performed to analyze the relationship between the thyroid function and eGFR. A multivariate generalized additive model (GAM) for Gaussian distributions was used to analyze the nonlinear relationship between the TSH and the eGFR, using the "mgcv" package in R developed by R Core Team. For the model fitting, Akaike information criterion, which indicates the more suitable model with a lower score, was calculated. Mediation analysis among fT4 and eGFR was performed using the "mediation" package. The treatment was fT4, the mediator was age, the outcome was eGFR, and the pretreatment covariates were age, sex, systolic and diastolic BP, TG, FG, HDL-C, body mass index, WC, hemoglobin, white blood cells, urine iodine, proteinuria, cancer, previous CV disease, liver disease, regular exercise and walking, and the status of income, education, smoking, alcohol consumption, and mental stress, with 2,000 times simulation. Using mediation analysis, average causal mediation effects, average direct effects, and total effects were calculated. A $p$ value $<0.05$ was considered statistically significant. All analyses were performed using R version 3.6.0 (R core Team 2019; R foundation for Statistical Computing, Vienna, Austria).

\section{Results}

The mean age of the 5,578 participants was 43.9 years and $49.5 \%$ were men. The percentages of participants with raised $\mathrm{BP}$, raised FG, raised TG, reduced HDL-C, and central obesity were $25.0,30.6,28.9,31.7$, and 32.1\%, respectively. Median TSH, mean fT4, and percentage of positive anti-TPO Abs were $2.2 \mathrm{mIU} / \mathrm{L}, 1.2 \mu \mathrm{g} / \mathrm{dL}$, and $6.6 \%$, respectively. The mean eGFR was $99.9 \mathrm{~mL} /$ $\min / 1.73 \mathrm{~m}^{2}$, and the percentage of subjects with proteinuria was $0.9 \%$.

The baseline characteristics of the study population, according to TSH quartiles, are shown in Table 1 . Although age $(p=0.745)$ was not associated with the TSH quartiles, the proportion of men $(p<0.001)$ decreased as the TSH quartile increased. The percentages of alcohol consumption $(p=0.011)$, current smoking $(p<0.001)$, and high mental stress $(p=$ $0.001)$ decreased, as the TSH quartile increased, while the percentage of high income ( $p=$ $0.005)$ increased. The percentage of raised FG $(p=0.022)$ decreased, while percentages of reduced HDL-C ( $p=0.005)$ and central obesity $(p=0.011)$ increased as the TSH quartile increased. As the TSH quartile increased, fT4 $(p<0.001)$ decreased, while urine iodine $(p<$ $0.001)$ and the percentage of positive anti-TPO Abs $(p<0.001)$ increased. With the increase of the TSH quartile, eGFR $(p<0.001)$ decreased. Proteinuria $(p=0.491)$ was not associated 
Kidney
Blood Pressure

Research
Kidney Blood Press Res 2020;45:442-454 DOI: $10.1159 / 000507290$

C 2020 The Author(s). Published by S. Karger AG, Basel www.karger.com/kbr

Kim et al.: Relationship Between Thyroid and Kidney Function

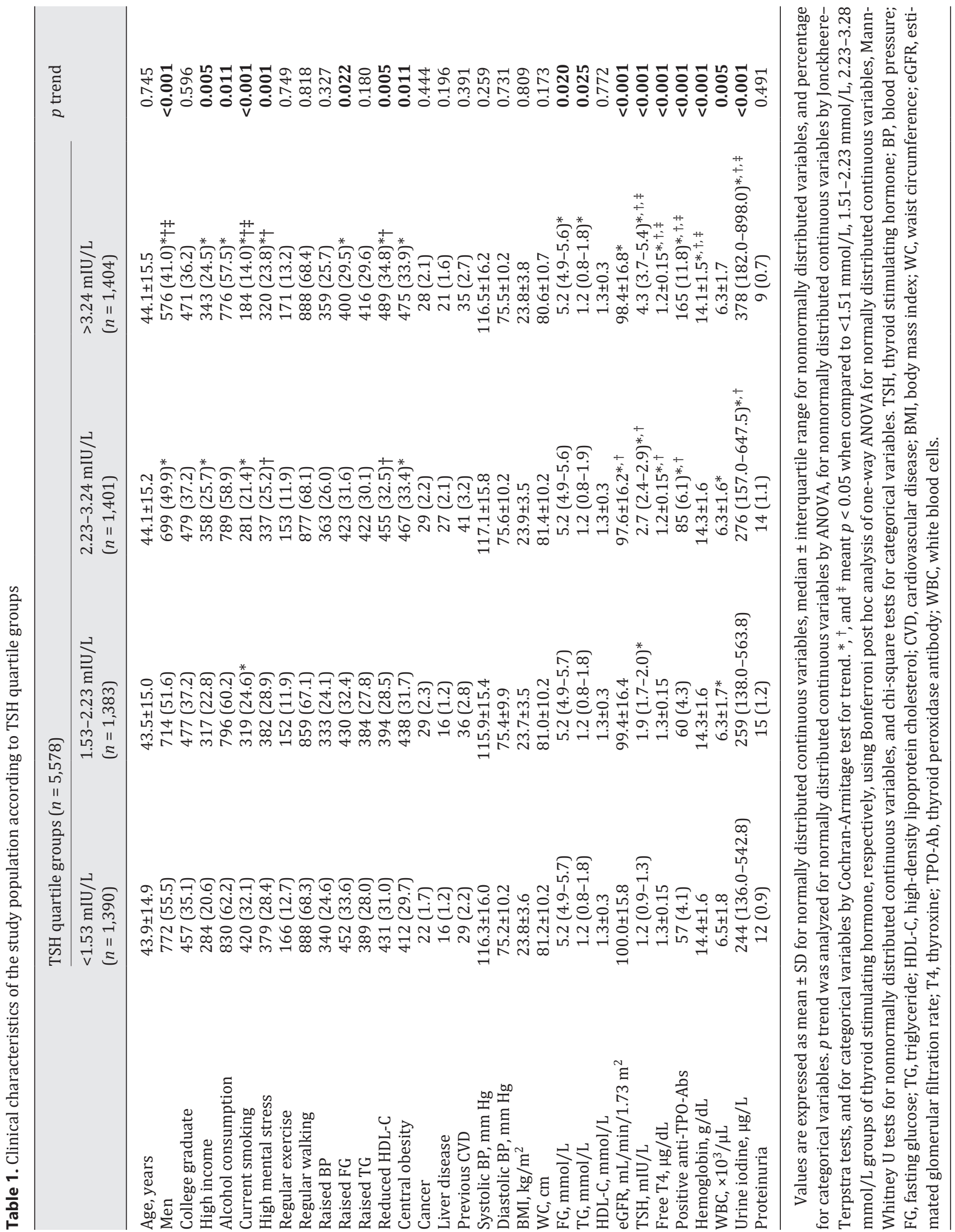


Table 2. The effect of thyroid function on kidney function

\begin{tabular}{|c|c|c|c|c|c|c|}
\hline & \multicolumn{2}{|l|}{ Univariate } & \multicolumn{2}{|l|}{ Model 1} & \multicolumn{2}{|l|}{ Model 2} \\
\hline & beta $(95 \% \mathrm{CI})$ & $p$ value & beta $(95 \% \mathrm{CI})$ & $p$ value & beta $(95 \% \mathrm{CI})$ & $p$ value \\
\hline TPO-Ab (yes vs. no) & $-3.8(-5.5$ to -2.1$)$ & $<0.001$ & $-2.5(-4.2$ to -0.9$)$ & & $-0.8(-2.1$ to 0.5$)$ & \\
\hline Log-TSH, mmol/L & $-1.5(-2.2$ to -0.8$)$ & $<0.001$ & $-1.5(-2.2$ to -0.8$)$ & $<0.001$ & $-1.8(-2.3$ to -1.2$)$ & $<0.001$ \\
\hline Free $\mathrm{T} 4, \mu \mathrm{g} / \mathrm{dL}$ & $3.9(1.1$ to 6.8$)$ & 0.007 & $2.9(0.0$ to 5.9$)$ & 0.052 & $-7.0(-9.4$ to -4.7$)$ & $<0.001$ \\
\hline
\end{tabular}

In model 1, adjusted beta and 95\% CI were calculated using multivariate linear regression analysis, entering all above variables with sex, systolic and diastolic blood pressure, triglyceride, fasting glucose, high-density lipoprotein cholesterol, body mass index, waist circumference, hemoglobin, urine iodine, white blood cells, proteinuria, cancer, previous cardiovascular disease, liver disease, regular exercise and walking, and the status of income, education, smoking, alcohol consumption, and mental stress status. In model 2, Adjusted beta and 95\% CI were calculated by adding age to the variables used in model 1 . TSH, thyroid stimulating hormone; T4, thyroxine; TPO-Ab, thyroid peroxidase antibody.

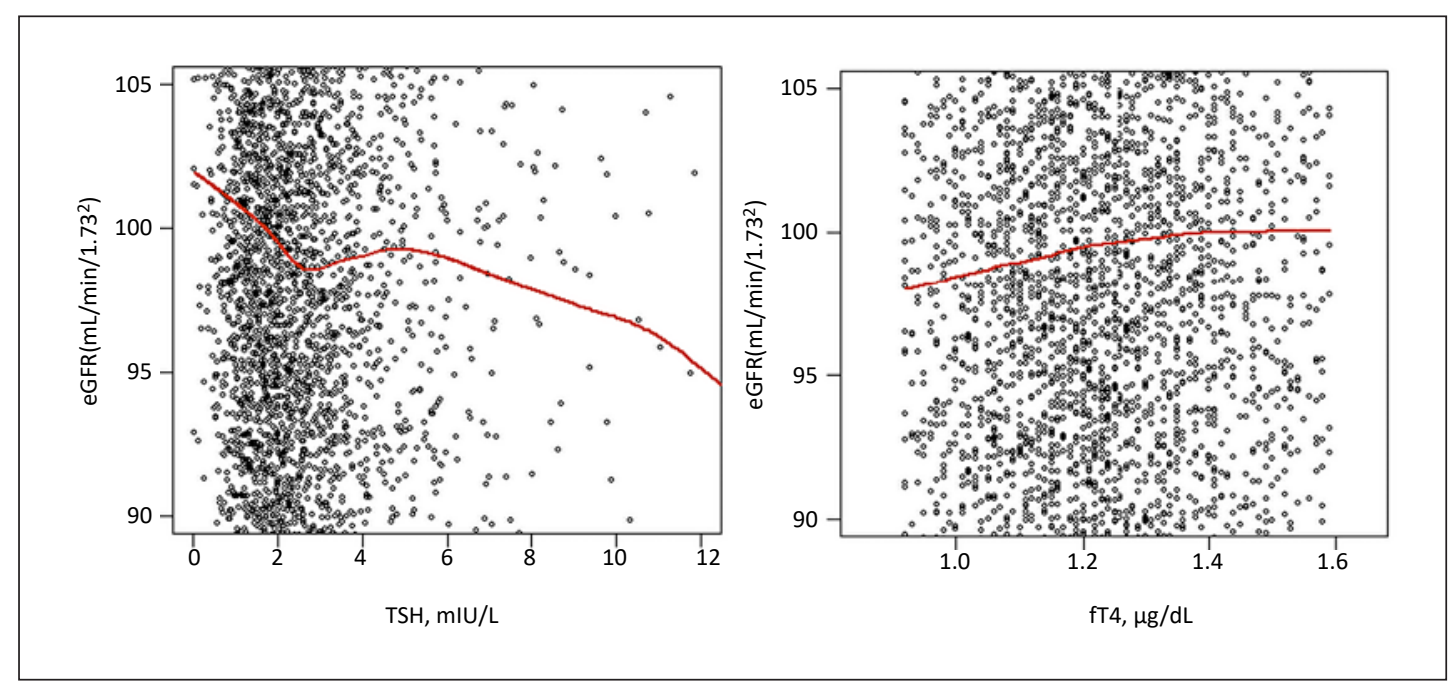

Fig. 1. Association between the TSH, fT4, and the eGFR. The red line indicated in the scatter plot is the LOWESS regression curve. All values were not transformed with either square root or logarithm. TSH, thyroid stimulation hormone; fT4, free thyroxine; eGFR, estimated glomerular filtration rate.

with the TSH quartile. White blood cell counts $(p=0.005)$ and the hemoglobin level $(p<$ 0.001 ) significantly decreased as the TSH quartile increased.

The LOWESS regression curve revealed that an increase in TSH was associated with a steady decrease in the eGFR (Fig. 1). Multivariable linear regression analysis confirmed this inverse relationship between TSH and eGFR (Table 2). In the GAM plots (Fig. 2), we found that the inverse relationship between TSH and eGFR was particularly evident when plasma TSH was $<4.0 \mathrm{mIU} / \mathrm{L}$; however, there was no definite cut-point identified using Akaike information criterion analysis. This prominent inverse relationship was also found in subgroup analysis, which demonstrated that the statistical significance between TSH and eGFR was present only in the non-hypothyroid groups (Fig. 3). Furthermore, there were no significant effect modifiers, including the status of anti-TPO Abs, urine iodine, and eGFR.

The LOWESS regression curve also revealed that an increase in fT4 was associated with an increase in the eGFR, plateauing in the higher fT4 range (Fig. 1). This positive relationship between fT4 and eGFR was confirmed in the univariate and multivariate linear regression 

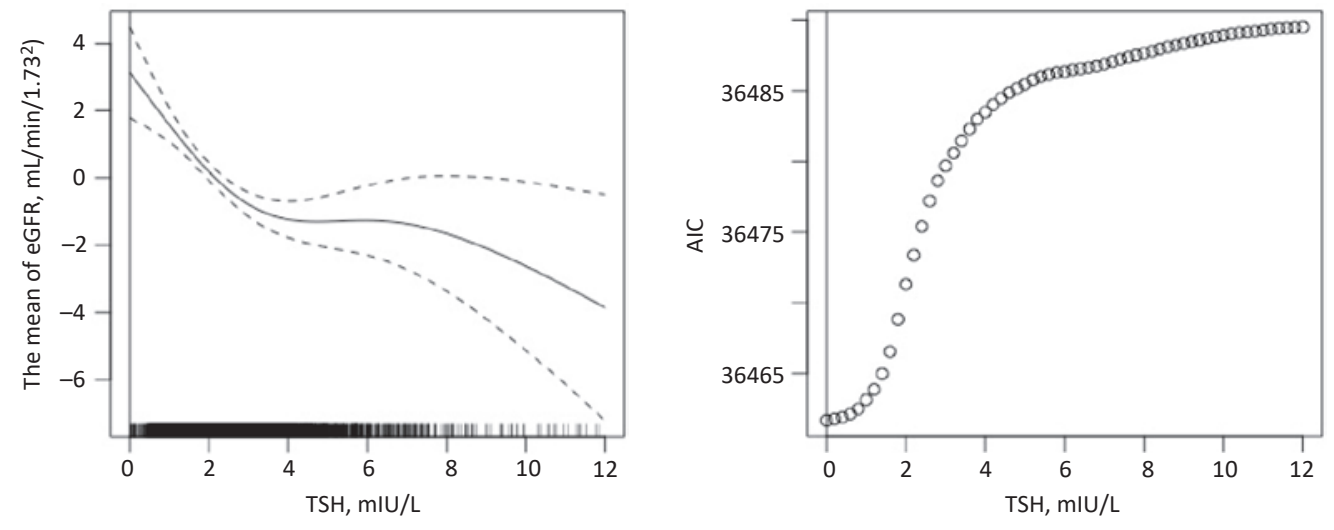

Fig. 2. GAM plot between TSH and eGFR. The dashed line indicate $95 \%$ CIs for value of the smoothed eGFR, using multivariate GAM analysis after adjusting for age, sex, systolic and diastolic BP, FG, TG, HDL-C, body mass index, WC, hemoglobin, urine iodine, white blood cells, proteinuria, cancer, previous CV disease, liver disease, regular exercise and walking, and the status of income, education, smoking, alcohol consumption, and mental stress. TSH, thyroid stimulating hormone; eGFR, estimated glomerular filtration rate; AIC, Akaike's information criterion.

\begin{tabular}{|c|c|c|c|}
\hline Subgroup No. of & people Adjusted & Beta $(95 \% \mathrm{Cl}, P)$ & Outcome: eGFR mL/min/1.732 \\
\hline \multirow{2}{*}{ Age } & $<43(n=2,677)$ & $-1.2(-2.0$ to $-0.4,0.005)$ & $\longmapsto$ \\
\hline & $\geq 43(n=2,901)$ & $-1.9(-2.7$ to $-1.1,<0.001)$ & $\longmapsto$ \\
\hline \multirow{2}{*}{ Sex } & Male $(n=2,761)$ & $-1.8(-2.6$ to $-1.0,<0.001)$ & $\longmapsto$ \\
\hline & Female $(n=2,817)$ & $-1.7(-2.3$ to $-1.0,<0.001)$ & $\longmapsto$ \\
\hline \multirow{2}{*}{ TSH } & $<4 \mathrm{mmol} / \mathrm{L}(n=4,744)$ & $-2.1(-2.8$ to $-1.4,<0.001)$ & $\longmapsto$ \\
\hline & $\geq 4 \mathrm{mmol} / \mathrm{L}(n=834)$ & $-1.9(-4.9$ to $1.1,0.222)$ & 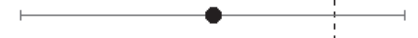 \\
\hline \multirow{2}{*}{ eGFR } & $<99 \mathrm{~mL} / \mathrm{min} / 1.73^{2}(n=2,793)$ & $-1.4(-2.1$ to $-0.7,<0.001)$ & $\longmapsto$ \\
\hline & $\geq 99 \mathrm{~mL} / \mathrm{min} / 1.73^{2}(n=2,785)$ & $-0.8(-1.2$ to $-0.4,<0.001)$ & $\bullet-1$ \\
\hline \multirow{2}{*}{ Anti-TPO Abs } & Negative $(n=5,211)$ & $-1.8(-2.3$ to $-1.2,<0.001)$ & $\longmapsto$ \\
\hline & Positive $(n=367)$ & $-1.9(-3.6$ to $-0.2,0.029)$ & $\bullet$ \\
\hline \multirow{2}{*}{ Urine iodine } & $<281 \mu \mathrm{g} / \mathrm{L}(n=2,607)$ & $-1.8(-2.6$ to $-1.0,<0.001)$ & $\longmapsto$ \\
\hline & $\geq 281 \mu \mathrm{g} / \mathrm{L}(n=2,613)$ & $-1.8(-2.5$ to $-1.1,<0.001)$ & $\longmapsto$ \\
\hline \multirow{3}{*}{ WBC } & $<6.1 \times 10^{3} / \mu \mathrm{L}(n=2,720)$ & $-2.1(-2.8$ to $-1.4,<0.001)$ & $\longmapsto$ \\
\hline & $\geq 6.1 \times 10^{3} / \mu \mathrm{L}(n=2,856)$ & $-1.5(-2.2$ to $-0.7,<0.001)$ & $\longmapsto$ \\
\hline & & & 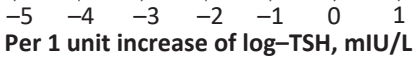 \\
\hline
\end{tabular}

Fig. 3. Subgroup analysis for the relationship between the TSH and the eGFR. Adjusted beta and 95\% CI were analyzed using multivariate linear regression analysis, entering into age, sex, systolic and diastolic BP, TG, FG, HDL-C, body mass index, WC, hemoglobin, white blood cells, urine iodine, proteinuria, cancer, previous $\mathrm{CV}$ disease, liver disease, regular exercise and walking, and the status of income, education, smoking, alcohol consumption, and mental stress status as covariates. TSH, thyroid stimulating hormone; eGFR, estimated glomerular filtration rate; WBC, white blood cells. 


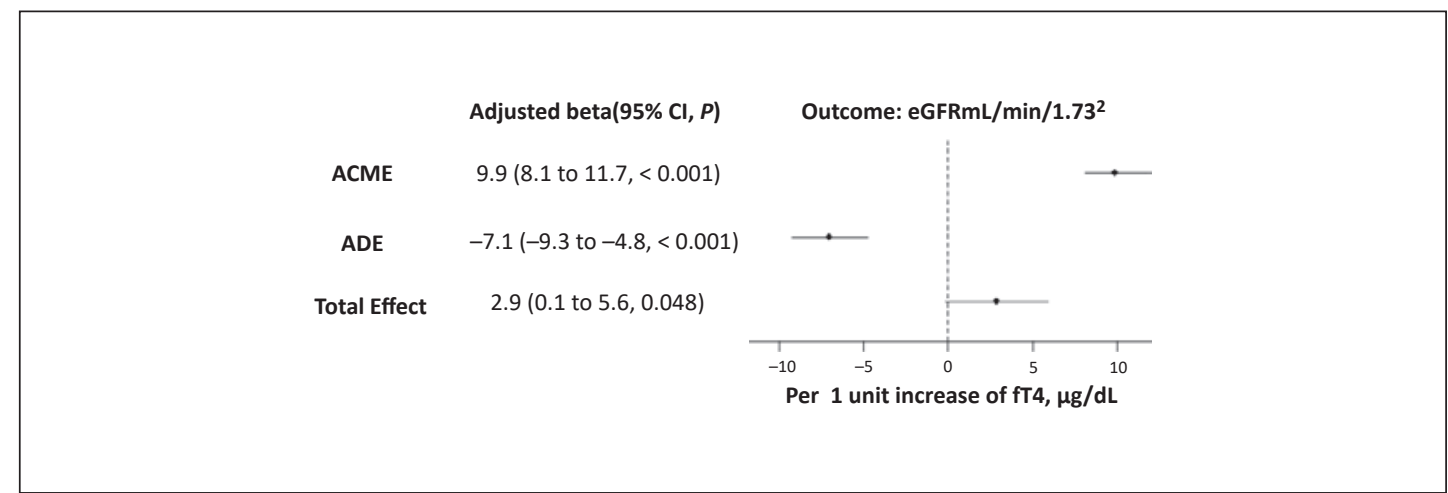

Fig. 4. Mediation analysis among fT4, age, and the eGFR. Outcome was eGFR, treatment was TSH, mediator was age, and pre-treatment covariates were age, sex, systolic and diastolic BP, TG, FG, HDL-C, body mass index, WC, hemoglobin, white blood cells, urine iodine, proteinuria, cancer, previous CV disease, liver disease, regular exercise and walking, and the status of income, education, smoking, alcohol consumption, and mental stress status. Overall effects of treatment on outcome were adjusted by mediator and pretreatment covariates using multivariate linear regression analysis. Mediator was modeled by treatment and pretreatment covariates using multivariate linear regression analysis. eGFR, estimated glomerular filtration rate; ACME, average causal mediation effect; ADE, average direct effect; fT4, free thyroxine.

Fig. 5. The hypothesis of pathogenetic link between thyroid function and kidney function. TSH, thyroid stimulating hormone; fT4, free thyroxine; T3, triiodothyronine; $\mathrm{TH}$, thyroid hormone; NTI, nonthyroidal illness; CKD, chronic kidney disease; eGFR, estimated glomerular filtration rate.

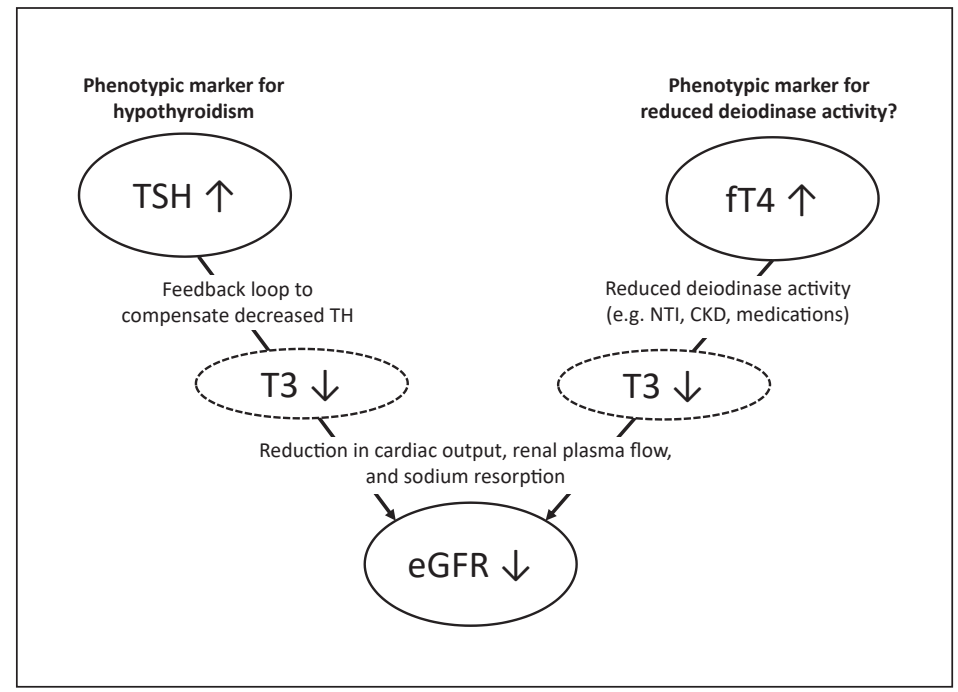

analyses (Model 1). However, adding age as covariate (Model 2) reversed the relationship between fT4 and eGFR (Table 2), suggesting a significant mediation effect by age. In mediation analysis (Fig. 4), increased fT4 was associated with increased eGFR indirectly when it was mediated by age. On the other hand, its direct effect on eGFR was negatively associated with eGFR. Finally, the total effect per $1 \mu \mathrm{g} / \mathrm{dL}$ increase of fT4 on eGFR was $2.9(0.1-5.6, p=0.048)$.

\section{Discussion/Conclusions}

SCH is common and about $15 \%$ of the elderly population may have SCH [40]. Despite its significant cardiometabolic hazards [11-17], treatment is only recommended when the TSH level is $\geq 10 \mathrm{mIU} / \mathrm{L}$ or if patients have significant symptoms [6]. However, kidney function is 


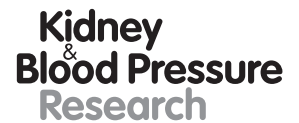

Kidney
Blood Pressure
Research \begin{tabular}{l|l}
\hline Kidney Blood Press Res 2020;45:442-454 \\
\hline DOI: 10.1159/000507290 & $\begin{array}{l}\text { @ 2020 The Author(s). Published by S. Karger AG, Basel } \\
\text { www.karger.com/kbr }\end{array}$ \\
\hline
\end{tabular}

Kim et al.: Relationship Between Thyroid and Kidney Function

not heavily considered when determining if treatment is necessary for $\mathrm{SCH}$; however, numerous studies have revealed that SCH may cause a decline in kidney function [20-24,30]. If small changes in the thyroid hormone levels, which are within the reference range, have negative effects on the kidney health, earlier replacement of thyroid hormone may prevent the progression of CKD in SCH patients [30]. Some studies have also reported that a higher TSH level within the reference range may also have renal hazards [25-27]; however, these studies did not evaluate a nonlinear relationship between TSH and eGFR. Although the metabolism of fT4 may be altered in CKD patients and a unique relationship between fT4 and eGFR may exist [19], an independent relationship between fT4 and eGFR has rarely been studied $[25,28,29]$. We simultaneously evaluated the impact of TSH and fT4 on eGFR, finding that TSH and eGFR showed an L-shaped relationship and demonstrated a significant association, particularly within the reference range of TSH. We also found that an increase in fT4 was directly associated with a decrease in eGFR, but was indirectly associated with increased eGFR via age.

In this study, we identified that an increased TSH was associated with decreased eGFR. These results were in accordance with previous studies. Chang et al. [20] reported that the OR and 95\% CI of SCH for CKD was 1.74 (1.18-2.56) after analyzing 74,356 subjects from Taiwan. Toda et al. [23] also suggested that the ORs (95\% CIs) of TSH 2.41-4.26 mIU/L and $>4.26 \mathrm{mIU} / \mathrm{L}$ for CKD were 1.49 (1.33-1.67, $p<0.001$ ) and 1.90 (1.57-2.30, $p<0.001)$, respectively, in 7,609 Japanese subjects. However, no known studies have investigated the nonlinear relationship between TSH and eGFR. In our GAM plots, we found that the relationship between TSH and eGFR was L-shaped, and the slope was steeper in the reference range for TSH (0-4 $\mathrm{mIU} / \mathrm{L}$ ). The subgroup analysis revealed that the renal hazard associated with increased TSH was only present in the non-hypothyroid groups. This may be because hypothyroidism is associated with increased age [41], bad habitual patterns [42, 43], lipotoxicity [44], and systemic inflammation [45], which may also attenuate thyroid hormone metabolism [46]. When considered with other studies that have reported an association between renal hazard and increased TSH, within the TSH reference range [22, 23, 25], our study results may suggest that a lower TSH level is associated with better kidney function. Therefore, future prospective studies need to be conducted to confirm the potential renoprotective effects of earlier thyroid hormone replacement.

We evaluated an independent relationship between fT4 and eGFR and identified that increased fT 4 was associated with decreased eGFR. To our knowledge, there have been 4 studies that have reported an independent relationship between fT4 and kidney function. Zhang et al. [25] reported that fT4, within the reference range, was not associated with incident CKD development (mean age $=38.0$ years). On the other hand, Huang et al. [28] suggested that increased fT4 was associated with an increased risk of incident CKD development (mean age $=59.3$ years). Similarly, Anderson et al. [47] reported a negative relationship between fT4 and creatinine clearance in a study of 2,180 subjects from the general population with normal thyroid function (mean age $=47.3$ years). Schultheiss et al. [29] also reported that increased fT 4 was associated with an increased risk for reduced kidney function (mean age $=57.4$ years). The differences in these results may partly be attributed to the age distribution of the participants; participants in 3 of the studies $[28,29,47]$ were much older than those in the other study [25]. In our study, the mean age was 43.9 years.

We further analyzed the independent effect of fT 4 on eGFR, with emphasis on the potential mediation effect of age. In multivariate linear regression analysis, adjusting for age reversed the relationship between fT 4 and eGFR. In mediation analysis, the direct effect (not affected by age) of increased fT4, within the reference range, was decreased eGFR. Based on these results, we hypothesized that increased fT4, within the reference range, may signify hypothyroidism in the tissue level; this was the case with TSH (Fig. 5). This may be because T4 is a 


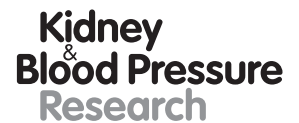

Kidney
Blood Pressure
Research

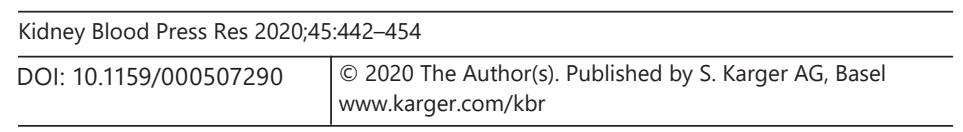

Kim et al.: Relationship Between Thyroid and Kidney Function

prohormone and has to be converted into triiodothyronine (T3) via deiodination to act on nuclear thyroid hormone receptors [48]. Decreased conversion to T3 due to reduced deiodinase activity results in increased T4 levels [48]. Therefore, increased fT4 levels, which may be associated with conditions of reduced deiodinase activity (including nonthyroidal illness) [49], CKD [19], and medications including amiodarone [48], may have a negative impact on eGFR. The effect of age on the relationship between fT 4 and eGFR, however, seemed to be more complex. In our mediation analysis, the indirect effect of increased fT4 levels, within the reference range, via age was to increase eGFR, although age is one of the known conditions to decrease deiodinase activity $[50,51]$. At this point, further investigation is necessary to delineate if an age-related increase of fT4 levels, within the reference range, is associated with functional hyperthyroidism at the tissue level [1].

Our study has some limitations that should be addressed. First, this is a cross-sectional study, so it cannot ascertain the causal relationship. Therefore, follow-up cohort studies are needed to confirm the effects that thyroid function had on kidney function. Second, the kidney function was estimated using a creatinine-based formula. Thyroid hormone status can affect the creatinine secretion of the kidney [52]. Therefore, studies using non-creatinine-based eGFR or measured GFR are needed to confirm our results. Third, TSH was measured once; therefore, laboratory error or a transient increase cannot be ruled out. Serum TSH values tend to return to normal more frequently in people with mild elevation (4-6 mIU/L) [53]. Forth, we speculated that the inverse relationship between fT 4 and eGFR was due to reduced conversion of T4 into T3 via decreased deiodination. Although this speculation can be convinced with the T3 level, T3 level was not measured in KNHANES. Therefore, subsequent studies that check both T3 and T4 need to be followed to confirm our speculation. Finally, the homogenous ethnicity of the patients limits the generalizability of this study. Despite these limitations, our study has several strengths. This is the first study to demonstrate a nonlinear relationship between thyroid function and kidney function. Also, we are the first to suggest that the mediation effect of age on the relationship between fT4 and eGFR may be different from that of other conditions. Third, the large number of participants and the decent participation rate may strengthen the results of our study. In the future, we believe that welldesigned prospective study about serial kidney function changes according to the thyroid hormone changes by replacement of thyroid hormone could provide a broader understanding of the relationship between thyroid hormone and kidney function.

In conclusion, increased TSH levels were associated with decreased eGFR, particularly in the reference range. The direct effect of increased fT4 levels, within the reference range, may be decreased eGFR; this suggests that functional hypothyroidism may be present at the tissue level, which was the case with TSH. Further studies need to be conducted to confirm the need for early replacement of thyroid hormones to protect kidney function.

\section{Acknowledgment}

There are no acknowledgments to declare.

\section{Statement of Ethics}

The study was conducted in accordance with the Declaration of Helsinki, and full approval of the study was obtained from the IRB of the Korea Centers for Disease Control (IRB 2013-07CON-03-4C, 2013-12EXP-03-5C, 2015-01-02-6C). All KNHANES participants provided written informed consent to have their data used in research. 


\section{Kidney \\ Blood Pressure Research}

\begin{tabular}{l|l}
\hline Kidney Blood Press Res 2020:45:442-454 \\
\hline DOI: 10.1159/000507290 & $\begin{array}{l}\text { @ 2020 The Author(s). Published by S. Karger AG, Basel } \\
\text { www.karger.com/kbr }\end{array}$ \\
\hline
\end{tabular}

Kim et al.: Relationship Between Thyroid and Kidney Function

\section{Disclosure Statement}

The authors have no conflicts of interest to declare.

\section{Funding Sources}

There are no funding sources to declare.

\section{Author Contributions}

S.W.L. conceptualization. S.H.K. data curation/formal analysis. H.K.M. investigation/ methodology. S.H.K. writing - original draft. H.K.M. writing - review and editing. S.W.L. supervision/validation. All authors read and approved the final manuscript.

\section{References}

1 Iglesias P, Bajo MA, Selgas R, Díez JJ. Thyroid dysfunction and kidney disease: an update. Rev Endocr Metab Disord. 2017 Mar;18(1):131-44.

2 Jonklaas J, Bianco AC, Bauer AJ, Burman KD, Cappola AR, Celi FS, et al.; American Thyroid Association Task Force on Thyroid Hormone Replacement. Guidelines for the treatment of hypothyroidism: prepared by the American thyroid association task force on thyroid hormone replacement. Thyroid. 2014 Dec;24(12):1670-751.

3 Ross DS, Burch HB, Cooper DS, Greenlee MC, Laurberg P, Maia AL, et al. 2016 American Thyroid Association guidelines for diagnosis and management of hyperthyroidism and other causes of thyrotoxicosis. Thyroid. 2016 Oct; $26(10): 1343-421$.

4 Ringel MD, Mazzaferri EL. Subclinical thyroid dysfunction-can there be a consensus about the consensus? J Clin Endocrinol Metab. 2005 Jan;90(1):588-90.

5 Cooper DS, Biondi B. Subclinical thyroid disease. Lancet. 2012 Mar;379(9821):1142-54.

6 Bekkering GE, Agoritsas T, Lytvyn L, Heen AF, Feller M, Moutzouri E, et al. Thyroid hormones treatment for subclinical hypothyroidism: a clinical practice guideline. BMJ. 2019 May;365:12006.

7 Meyerovitch J, Rotman-Pikielny P, Sherf M, Battat E, Levy Y, Surks MI. Serum thyrotropin measurements in the community: five-year follow-up in a large network of primary care physicians. Arch Intern Med. 2007 Jul; 167(14):1533-8.

8 Li Y, Teng D, Shan Z, Teng X, Guan H, Yu X, et al. Antithyroperoxidase and antithyroglobulin antibodies in a five-year follow-up survey of populations with different iodine intakes. J Clin Endocrinol Metab. 2008 May; 93(5):1751-7.

9 Brenta G, Mutti LA, Schnitman M, Fretes O, Perrone A, Matute ML. Assessment of left ventricular diastolic function by radionuclide ventriculography at rest and exercise in subclinical hypothyroidism, and its response to L-thyroxine therapy. Am J Cardiol. 2003 Jun;91(11):1327-30.

10 Taddei S, Caraccio N, Virdis A, Dardano A, Versari D, Ghiadoni L, et al. Impaired endothelium-dependent vasodilatation in subclinical hypothyroidism: beneficial effect of levothyroxine therapy. J Clin Endocrinol Metab. 2003 Aug;88(8):3731-7.

11 Liu D, Jiang F, Shan Z, Wang B, Wang J, Lai Y, et al. A cross-sectional survey of relationship between serum TSH level and blood pressure. J Hum Hypertens. 2010 Feb;24(2):134-8.

12 Danese MD, Ladenson PW, Meinert CL, Powe NR. Clinical review 115: effect of thyroxine therapy on serum lipoproteins in patients with mild thyroid failure: a quantitative review of the literature. J Clin Endocrinol Metab. 2000 Sep;85(9):2993-3001.

13 Maratou E, Hadjidakis DJ, Kollias A, Tsegka K, Peppa M, Alevizaki M, et al. Studies of insulin resistance in patients with clinical and subclinical hypothyroidism. Eur J Endocrinol. 2009 May;160(5):785-90.

14 Rodondi N, Newman AB, Vittinghoff E, de Rekeneire N, Satterfield S, Harris TB, et al. Subclinical hypothyroidism and the risk of heart failure, other cardiovascular events, and death. Arch Intern Med. 2005 Nov; 165(21):2460-6.

15 Rodondi N, Bauer DC, Cappola AR, Cornuz J, Robbins J, Fried LP, et al. Subclinical thyroid dysfunction, cardiac function, and the risk of heart failure. The Cardiovascular Health study. J Am Coll Cardiol. 2008 Sep;52(14): 1152-9.

16 Razvi S, Weaver JU, Vanderpump MP, Pearce SH. The incidence of ischemic heart disease and mortality in people with subclinical hypothyroidism: reanalysis of the Whickham Survey cohort. J Clin Endocrinol Metab. 2010 Apr;95(4):1734-40. 
Kim et al.: Relationship Between Thyroid and Kidney Function

17 Walsh JP, Bremner AP, Bulsara MK, O’Leary P, Leedman PJ, Feddema P, et al. Subclinical thyroid dysfunction as a risk factor for cardiovascular disease. Arch Intern Med. 2005 Nov;165(21):2467-72.

18 Falk SA, Buric V, Hammond WS, Conger JD. Serial glomerular and tubular dynamics in thyroidectomized rats with remnant kidneys. Am J Kidney Dis. 1991 Feb;17(2):218-27.

19 Mariani LH, Berns JS. The renal manifestations of thyroid disease. J Am Soc Nephrol. 2012 Jan;23(1):22-6.

20 Chang YC, Chang CH, Yeh YC, Chuang LM, Tu YK. Subclinical and overt hypothyroidism is associated with reduced glomerular filtration rate and proteinuria: a large cross-sectional population study. Sci Rep. 2018 Feb;8(1):2031.

21 Zhang Y, Wang Y, Tao XJ, Li Q, Li FF, Lee KO, et al. Relationship between Thyroid Function and Kidney Function in Patients with Type 2 Diabetes. Int J Endocrinol. 2018 Nov;2018:1871530.

22 Peixoto de Miranda ÉJ, Bittencourt MS, Goulart AC, Santos IS, de Oliveira Titan SM, Ladeira RM, et al. Thyrotropin levels are associated with chronic kidney disease among healthy subjects in cross-sectional analysis of the Brazilian Longitudinal Study of Adult Health (ELSA-Brasil). Clin Exp Nephrol. 2017 Dec;21(6):1035-43.

23 Toda A, Hara S, Kato M, Tsuji H, Arase Y. Association of Thyrotropin Concentration with Chronic Kidney Disease in a Japanese General Population Cohort. Nephron. 2019;142(2):91-7.

24 Chuang MH, Liao KM, Hung YM, Wang PY, Chou YC, Chou P. Abnormal Thyroid-Stimulating Hormone and Chronic Kidney Disease in Elderly Adults in Taipei City. J Am Geriatr Soc. 2016 Jun;64(6):1267-73.

25 Zhang Y, Chang Y, Ryu S, Cho J, Lee WY, Rhee EJ, et al. Thyroid hormone levels and incident chronic kidney disease in euthyroid individuals: the Kangbuk Samsung Health Study. Int J Epidemiol. 2014 Oct;43(5):162432.

26 Asvold BO, Bjøro T, Vatten LJ. Association of thyroid function with estimated glomerular filtration rate in a population-based study: the HUNT study. Eur J Endocrinol. 2011 Jan;164(1):101-5.

27 Sun MT, Hsiao FC, Su SC, Pei D, Hung YJ. Thyrotropin as an independent factor of renal function and chronic kidney disease in normoglycemic euthyroid adults. Endocr Res. 2012;37(3):110-6.

28 Huang X, Ding L, Peng K, Lin L, Wang T, Zhao Z, et al. Thyroid hormones associate with risk of incident chronic kidney disease and rapid decline in renal function: a prospective investigation. J Transl Med. 2016 Dec;14(1): 336.

29 Schultheiss UT, Daya N, Grams ME, Seufert J, Steffes M, Coresh J, et al. Thyroid function, reduced kidney function and incident chronic kidney disease in a community-based population: the Atherosclerosis Risk in Communities study. Nephrol Dial Transplant. 2017 Nov;32(11):1874-81.

30 Shin DH, Lee MJ, Kim SJ, Oh HJ, Kim HR, Han JH, et al. Preservation of renal function by thyroid hormone replacement therapy in chronic kidney disease patients with subclinical hypothyroidism. J Clin Endocrinol Metab. 2012 Aug;97(8):2732-40.

31 Bensenor IM, Olmos RD, Lotufo PA. Hypothyroidism in the elderly: diagnosis and management. Clin Interv Aging. 2012;7:97-111.

32 Hadlow NC, Rothacker KM, Wardrop R, Brown SJ, Lim EM, Walsh JP. The relationship between TSH and free $\mathrm{T}_{4}$ in a large population is complex and nonlinear and differs by age and sex. J Clin Endocrinol Metab. $2013 \mathrm{Jul}$; 98(7):2936-43.

33 Rosario PW. Natural history of subclinical hyperthyroidism in elderly patients with TSH between 0.1 and 0.4 mIU/l: a prospective study. Clin Endocrinol (Oxf). 2010 May; 72(5):685-8.

34 Surks MI, Boucai L. Age- and race-based serum thyrotropin reference limits. J Clin Endocrinol Metab. 2010 Feb;95(2):496-502.

35 Jang J, Kim Y, Shin J, Lee SA, Choi Y, Park EC. Association between thyroid hormones and the components of metabolic syndrome. BMC Endocr Disord. 2018 May;18(1):29.

36 Oshiro I, Takenaka T, Maeda J. New method for hemoglobin determination by using sodium lauryl sulfate (SLS). Clin Biochem. 1982 Apr;15(2):83-8.

37 Delanghe JR, Speeckaert MM. Creatinine determination according to Jaffe-what does it stand for? NDT Plus. 2011 Apr;4(2):83-6.

38 Levey AS, Stevens LA, Schmid CH, Zhang YL, Castro AF 3rd, Feldman HI, et al.; CKD-EPI (Chronic Kidney Disease Epidemiology Collaboration). A new equation to estimate glomerular filtration rate. Ann Intern Med. 2009 May;150(9):604-12.

39 Alberti KG, Zimmet P, Shaw J. Metabolic syndrome-a new world-wide definition. A Consensus Statement from the International Diabetes Federation. Diabet Med. 2006 May;23(5):469-80.

40 Fatourechi V. Subclinical hypothyroidism: an update for primary care physicians. Mayo Clin Proc. 2009;84(1): 65-71.

41 Aggarwal N, Razvi S. Thyroid and aging or the aging thyroid? An evidence-based analysis of the literature. J Thyroid Res. 2013;2013:481287.

42 Masaki M, Koide K, Goda A, Miyazaki A, Masuyama T, Koshiba M. Effect of acute aerobic exercise on arterial stiffness and thyroid-stimulating hormone in subclinical hypothyroidism. Heart Vessels. 2019 Aug;34(8): 1309-16.

43 Nyström E, Bengtsson C, Lapidus L, Petersen K, Lindstedt G. Smoking-a risk factor for hypothyroidism. J Endocrinol Invest. $1993 \mathrm{Feb}$;6(2):129-31.

44 Zhao M, Tang X, Yang T, Zhang B, Guan Q, Shao S, et al. Lipotoxicity, a potential risk factor for the increasing prevalence of subclinical hypothyroidism? J Clin Endocrinol Metab. 2015 May;100(5):1887-94. 
45 Enia G, Panuccio V, Cutrupi S, Pizzini P, Tripepi G, Mallamaci F, et al. Subclinical hypothyroidism is linked to micro-inflammation and predicts death in continuous ambulatory peritoneal dialysis. Nephrol Dial Transplant. 2007 Feb;22(2):538-44.

46 Kelly GS. Peripheral metabolism of thyroid hormones: a review. Altern Med Rev. 2000 Aug;5(4):306-33.

47 Anderson JL, Gruppen EG, van Tienhoven-Wind L, Eisenga MF, de Vries H, Gansevoort RT, et al. Glomerular filtration rate is associated with free triiodothyronine in euthyroid subjects: comparison between various equations to estimate renal function and creatinine clearance. Eur J Intern Med. 2018 Feb;48:94-9.

48 Bianco AC, Kim BW. Deiodinases: implications of the local control of thyroid hormone action. J Clin Invest. 2006 Oct;116(10):2571-9.

49 Warner MH, Beckett GJ. Mechanisms behind the non-thyroidal illness syndrome: an update. J Endocrinol. 2010 Apr;205(1):1-13.

50 Franceschi C, Ostan R, Mariotti S, Monti D, Vitale G. The Aging Thyroid: A Reappraisal Within the Geroscience Integrated Perspective. Endocr Rev. 2019 Oct;40(5):1250-70.

51 Mariotti S, Franceschi C, Cossarizza A, Pinchera A. The aging thyroid. Endocr Rev. 1995 Dec;16(6):686-715.

52 Kreisman SH, Hennessey JV. Consistent reversible elevations of serum creatinine levels in severe hypothyroidism. Arch Intern Med. 1999 Jan;159(1):79-82.

53 Díez JJ, Iglesias P, Burman KD. Spontaneous normalization of thyrotropin concentrations in patients with subclinical hypothyroidism. J Clin Endocrinol Metab. 2005 Jul;90(7):4124-7. 